\title{
Scientific and ethical consequences of disease prediction
}

\author{
Work group chairman: M. Siegler ${ }^{1}$ \\ Report prepared by: S. Amiel ${ }^{2}$ and J. Lantos ${ }^{3}$ \\ ${ }^{1}$ Center for Clinical Medical Ethics, University of Chicago, Chicago, Illinois, USA; and ${ }^{2}$ Unit for Metabolic Medicine, Guy's Hospital, \\ London, UK; and ${ }^{3}$ La Rabida Hospital, Chicago, Illinois, USA
}

Summary. HLA-typing, gene analysis, anti-islet cell antibody testing and metabolic studies can identify people at high risk for developing Type 1 (insulin-dependent) diabetes mellitus prior to the onset of clinical disease. The positive predictive value of these tests is high in first degree relatives of patients with Type 1 diabetes, but six times less so in the general population, where disease incidence is much lower but where $90 \%$ of new cases occur. Multiple testing improves sensitivity but decreases specificity. Intervention strategies are being designed with the aim of delaying or preventing progression to clinical disease. The more invasive the intervention, the greater is the specificity required. The practical and ethical implications of identifying high risk of diabetes in healthy individuals are complex and require further research, but some lessons can be learned from the experience of other disease prediction programmes.

Key words: Disease prediction, disease prevention, ethics.

\section{Introduction}

Advances in both immunologic and genetic tests may soon make it possible to predict whether some individuals will develop Type 1 (insulin-dependent) diabetes mellitus. Advances in therapeutics also may make it possible to prevent or delay the development of Type 1 diabetes. These exciting prospects create enthusiasm for clinical trials to demonstrate the benefits of presymptomatic diagnosis or preventive treatment. The enthusiasm of the diabetes community should be tempered, however, by the realisation that attempts to develop presymptomatic tests and preventive interventions for other diseases have raised difficult clinical and ethical issues [1].

Presymptomatic testing, which generally provides only information about health risks, should be contrasted with the routine diagnosis of a symptomatic disease, which generally provides direct patient benefits. These patient benefits may include not only information about health risks, but also specific treatment, relief of symptoms, improvement of function, and, in the best cases, cure.

Presymptomatic testing programmes, such as newborn screening for metabolic diseases and cystic fibrosis, and screening of adults for sickle cell disease and Huntington's disease, have not always provided benefits to those screened and sometimes have resulted in unanticipated or undesirable consequences. These diseases are similar to Type 1 diabetes in that they are chronic, incurable, and cannot be prevented from occurring at the present time.

Presymptomatic diagnosis of chronic diseases such as Type 1 diabetes, which cannot now be prevented, is even more controversial. For some people, predictive information itself may be of value, even when not associated with tangible health benefits. Others find the predictive information that labels them with a disease to be either useless or actually harmful. Furthermore, such information may result in social disadvantages, such as loss of employment, difficulty in obtaining health or life insurance [2], and difficulty in obtaining a home mortgage loan. Presymptomatic diagnosis of Type 1 diabetes, in the absence of effective preventive therapy, may be associated with some of these negative implications. Unless disease prediction can be coupled with an efficacious prevention strategy or early treatment programme, the positive consequences of disease prediction must be very carefully balanced against the inevitable negative consequences.

In the case of Type 1 diabetes, how should we assess the advantages and disadvantages of early prediction? The risk-benefit ratio must be calculated both in terms of possible direct effects on the individual involved and possible indirect effects, i.e. those on other individuals or society as a whole. Each stage of the predictive pathway must be examined. What are the risks and benefits of the diagnostic procedures themselves? How sensitive and specific are the tests? Will the knowledge that a person has a high risk of developing a chronic disabling disease cause 
prolonged anxiety or affect employment, marriage, or financial prospects? Will the knowledge that this disease is likely to occur in the future allow early therapeutic interventions that would not be effective at a later time and that may avert or attenuate the disease?

In this report, we examine the current status of the feasibility of predicting who will develop Type 1 diabetes; we briefly comment on the possibility of therapeutic intervention in the presymptomatic stage of diabetes (see "Treatment of Diabetes Mellitus"); we note the cautionary lessons that may be learned from earlier screening efforts in other chronic diseases; we discuss the ethical and psychosocial issues that arise in presymptomatic testing in Type 1 diabetes; and we conclude by offering some recommendations for presymptomatic screening for Type 1 diabetes.

\section{State-of-the-art of prediction}

\section{Disease prediction for direct patient benefit}

Unless preventive strategies are entirely benign and inexpensive, efforts to prevent Type 1 diabetes should initially be directed towards individuals at high risk of developing the disease. Equally, the efficacy of any such strategy can best be assessed if the strategy is applied to a group of individuals with a high rate of progression to diabetes in the absence of any intervention. Thus accurate prediction of Type 1 diabetes becomes essential.

The development of Type 1 diabetes in an individual is a slow, stepped progression which depends upon genetic susceptibility, environmental influences and immunological activation, culminating in loss of pancreatic Beta-cell function [3, 4]. To some extent, progression through these preclinical stages is predictable and the identification of each stage in an individual gives clues to his or her risk of eventually developing clinical disease. Genetic tests, for example, can only determine predisposition, whereas a combination of genetic markers and other markers of immune activation, insulitis and decreased Beta-cell mass may define a true presymptomatic phase of Type 1 diabetes itself. This distinction between predisposition to Type 1 diabetes and presymptomatic disease has important implications for the design of potential interventional strategies.

Type 1 diabetes arises as a result of immune-mediated destruction of pancreatic Beta cells. Its clinical manifestations develop only slowly - there is a long prodromal phase (which is probably longer in adults than in children) in which evidence of immune activation can be found [3, 4]. Mild abnormalities of insulin secretion or glucose homeostasis or both can then be identified before symptomatic diabetes is demonstrable $[5,6]$. This slow development of clinical disease potentially admits several opportunities for intervention in the disease process to retard or arrest its progression. By the time the disease is clinically manifest, it is likely that the destructive process is too advanced for satisfactory curative intervention. It is therefore essential to identify earlier stages to permit earlier, preventive intervention.

Genetic factors in Type 1 diabetes. Genetic susceptibility to Type 1 diabetes is inherited from the parents. In recent years the possession of certain gene alleles has been associated with high or low risk of developing diabetes [5]. It is important to realise that these genetic markers can only indicate a predisposition to Type 1 diabetes and do not constitute a presymptomatic diagnosis.

The "diabetes-related" genomes are not inherited in simple Mendelian fashion. The very high prevalence of "diabetogenic" genes in the total population suggests that they are preferentially inherited. It is suggested that they may convey some as yet unknown survival benefit, e.g., resistance to infection. Furthermore, the risk of inheritance of a given allele depends upon which parent carries it children of fathers with Type 1 diabetes carry approximately an $8 \%$ risk of developing Type 1 diabetes themselves compared with a risk of only $2 \%$ in children of diabetic mothers.

The histocompatibility complex has been implicated in the pathogenesis of Type 1 diabetes in both human and animal models. It is likely that expression of certain HLA types on membranes of cells involved in the immune process renders the individual more susceptible to develop the disease. HLA DR3 and DR4 are found with high frequency in Type 1 diabetic subjects (for Type 1 diabetes, $55.8 \%$ of the population of the United States have DR3 vs $22.8 \%$ of the non-diabetic background population, and $67.3 \%$ vs $25.2 \%$ have DR4, respectively). In siblings of children with diabetes, HLA identity with the proband is associated with a risk of developing diabetes that is 15$18 \%$. Sharing one HLA allele with the affected proband (haploidentity) creates a risk for diabetes of $7-8 \%$, while possession of two totally different HLA alleles confers a risk hardly greater than that of the normal population.

Further investigation has identified the closely associated DQ $\beta$ locus as the important site at which a change can result in susceptibility to Type 1 diabetes, perhaps three times more powerfully associated with diabetes than the DR locus itself, which is more probably a modifier of the action of the DQ locus. In particular, the loss of the amino acid residue aspartate (Asp) at position 57 on the DQ $\beta$ molecule creates a special susceptibility [7]. Ninety-six percent of individuals with Type 1 diabetes are homozygous for non-Asp 57, compared with $19.5 \%$ of the general population, and only $4 \%$ are heterozygous compared with $80.5 \%$ of non-diabetic control subjects. Although initial investigation suggested a very close correlation between the frequency of non-Asp 57, homozygosity and the incidence of Type 1 diabetes, examination of additional populations has weakened the association. A further factor under investigation which may also confer susceptibility to Type 1 diabetes is the presence of an arginine residue at position 52 of the DQ $\alpha$ molecule. 
It now seems likely that the whole HLA haplotype is important in determining susceptibility to Type 1 diabetes. Non-MHC genes are also implicated, including genes on chromosome 11 near the insulin gene. If the situation in humans is similar to that of the NOD mouse, five or six such genes are likely to be involved.

In families with a diabetic proband, siblings have an overall risk of $6.9 \%$ and children of the proband a $5.6 \%$ risk of developing Type 1 diabetes themselves. Genetic studies can lead to more accurate prediction of disease risk but remain only a weak predictor of disease. Even in genetically identical twins, where one twin develops Type 1 diabetes, the other twin has only a one in three risk of also developing Type 1 diabetes within the next 10 years or indeed thereafter. Furthermore, the "diabetogenic" HLA types occur quite frequently in non-diabetic populations. Even homozygosity for non-Asp 57 is only weakly predictive, occurring in approximately $20 \%$ of non-diabetic individuals. The predictive value of genetic screening is even less outside the context of families with a diabetic proband. The genotype DR4 DQW6/DR3 DQW2 carries only an $8.5 \%$ risk for Type 1 diabetes, heterozygosity for DR3/DR4 3\% and homozygosity for non-Asp 57 a $2.5 \%$ risk for Type 1 diabetes in such diabetes-unrelated individuals $[7,8]$.

Environmental influences. The lack of complete concordance for the development of Type 1 diabetes between genetically identical twins confirms that genetic factors alone do not account for the disease. There is other evidence pointing to a substantial environmental influence on the pathogenesis of Type 1 diabetes. For example, the geographic distribution of incidence rates for Type 1 diabetes across Europe cannot be explained on the basis of genetic variation alone - the differences are too great. There is a north-south gradient, with more than 20 new cases per 100,000 population in Scandinavia and Finland, between 10 and 19 new cases per 100,000 population in countries such as the United Kingdom, Denmark and Belgium, and only four to nine new cases per 100,000 population in southern countries such as Spain, Italy and Greece. Only Sardinia, with its unexpectedly (and unexplained) high incidence of Type 1 diabetes lies outside this pattern. Genetic variation (in terms of known genetic markers) could explain a proportion of these differences.

Phase of immune activation and insulitis. At present we do not have the ability to use genetic markers accurately to identify individuals at high risk for development of Type 1 diabetes. Nor do we understand the environmental factors that initiate the immune-mediated destruction of the Beta cell in genetically sensitive individuals. Attempts to identify markers of high risk of progression to Type 1 diabetes have thus turned to the next stage in the pathogenesis of the disease and sought to identify markers of early activation of the immune response. The best validated markers are islet cell antibodies (ICA), markers which stain all cell types in the islet and are measured semi-quantitatively by a fluorescent antibody technique. The Juvenile Diabetes Foundation International has supported the establishment of standards for measuring ICA so that data can be pooled from various laboratories. ICA are quantitatively expressed now in international (JDF) units.

Consensus on the prognostic significance of ICA is emerging. It seems clear that, whether the appearance of ICA in the circulation is a primary event in the destruction of the Beta cell or just a marker that such destruction is occurring, the presence of significant titre of ICA (e.g. over $20 \mathrm{JDF}$ units) may be a valuable marker for very early diabetic disease. Again this issue has been examined in the relatively high risk population comprising the first degree relatives of probands with Type 1 diabetes. In 719 such individuals in the Bart's-Windsor/Bart's-Oxford Study, 2 of 679 ICA negative subjects have developed Type I diabetes compared with 16 of 40 ICA positive subjects [9]. Data from Gainesville agree - with $70 \%$ of people with ICA levels in excess of $20 \mathrm{JDF}$ units developing Type 1 diabetes, $35 \%$ within 5 years of detection [10]. A few false positives may account for the $10-30 \%$ of ICA positive subjects who do not progress to Type 1 diabetes. Differential staining properties of ICA may help to identify such "non-progressors" in the future.

New immune markers are being described which may add to the sensitivity of the test. Antibodies against subtypes of ICA and against GAD are described. But currently ICA can and are being used as the chief basis of disease prediction.

A further issue is the timing and permanence of conversion to ICA positivity in an individual subject. Type 1 diabetes, although it can arise in any age group, is primarily a disease of youth. One-third of Type 1 diabetes patients are diagnosed before they are 10 years old, onethird between ages 10 and 20 and one-third when they are over 20 years. In the Bart's-Oxford Family Study, $25 \%$ of probands were diagnosed before they were 5 years old. In a study by Elliott and colleagues from New Zealand, the prevalence of Type 1 diabetes did not alter across the age groups, but seroconversion to ICA positivity (using a highly sensitive assay) was maximal in the age group 5 years and younger. Ultimately, screening will have to be done on very young children. It is relevant that children seem to have a faster rate of Beta-cell loss and a shorter prodrome than individuals developing the disease in adult life. This has implications for the design of research studies, which are more likely to show positive effects, if any, in younger subjects.

In planning for the future, a major research effort has begun to establish an international register of people with positive ICA test results. In line with the recommendations of the American Diabetes Association Policy Statements of 1990, ICARUS (the ICA Register of Users Study) has set up standardised methods of study of "prediabetes." It organises reference laboratories, provides a 
mechanism for sharing data, and acts as a clearing house for collaborative research and a repository for both data and storage of sera and cells for future projects.

Phase of Beta-cell destruction-presymptomatic disease. The possession of high titre ICA indicates a relatively high risk of future Type 1 diabetes in first degree relatives of diabetic probands, but the Bart's-Oxford and Gainesville experience described above still has only about $35 \%$ of subjects diagnosed at 5 years. The Joslin group has devised a formula for calculating the risk of and time to progression of clinical Type 1 diabetes on the basis of high titre ICA, the quantitation of anti-insulin antibodies and the first phase insulin response to intravenous glucose challenge (this is the sum of the insulin levels at 1 and 3 minutes after controlled administration of an intravenous glucose load) [11].

This model moves towards the elimination of false negatives. It is claimed that $100 \%$ of subjects whose predicted time to clinical diabetes is calculated to be less than 3 years will have developed the disease within 4 . This predictive test can be used to identify first degree relatives in whom to test potential preventive interventions in small studies of manageable duration. However, it is important to remember that the predictive tests, developed in first degree relatives of probands with Type 1 diabetes, will only identify perhaps $3-5 \%$ of potential victims of Type 1 diabetes. Ninety percent of new cases arise in people without a family history. Furthermore, the ICA test is highly sensitive. Only $50 \%$ of potential subjects will have titres sufficient to justify the application of the three parameter model. Finally, it should be noted that the first phase insulin response to the intravenous glucose tolerance test shows considerable intra-individual variability, although subjects with total loss of this response are unlikely to regain it in the natural history of the disease.

The importance of using predictive tests of high specificity will be discussed later. At this point, it should however be emphasised that the more invasive the planned intervention, the more specific the predictive tests must be. An innocuous intervention could be planned using a less specific test, so that individuals incorrectly identified as having high risk will not have been inconvenienced by the intervention. This would not be true for a more toxic intervention. A vital principle is illustrated here. In planning preventive strategies, the predictive models cannot be meaningfully discussed without consideration of the nature of the planned intervention.

There is another disadvantage of the dual parameter model when it comes to be applied to the planning of interventional strategies. It is most effective in predicting onset of clinical disease within the relatively near future, when the first phase insulin response is already low. This is probably a relatively late stage in the progressive onset of Type 1 diabetes, when substantial damage has already been done. It is much less sensitive for earlier stages of disease. This means the test is useful for the planning of pilot studies but will perhaps be less useful at the stage of expanding such studies into clinical practice in the future.

Screening for markers of prediabetes in the general population. What is the importance of ICA in the population as a whole? Approximately 3,000 healthy schoolchildren have been screened for ICA in the Oxford region in England - a population in whom the ascertainment of new cases of Type 1 diabetes is greater than $95 \%$. In these non-diabetic related children, there was a prevalence of ICA, positivity of $1.5 \%(0.8 \%$ having a titre of more than $20 \mathrm{JDF}$ units), while the prevalence of Type 1 diabetes is low (around $0.8 \%$ at age 20) [12]. In first degree relatives of Type 1 diabetic probands in the same geographical area (the Bart's-Oxford Study), there was a $6.5 \%$ prevalence of ICA positivity in siblings, with a prevalence of Type 1 diabetes of as much as $5 \%$ by age 20 . As will be discussed later, Bayes' rule implies that the positive predictive value of a test depends upon the prevalence of the disease in the population tested. Thus ICA will have high predictive value in a population of first degree relatives of patients with Type 1 diabetes, in whom the prevalence of Type 1 diabetes is relatively high, but will be less useful in the general population in whom most new disease will arise. There is a six-fold difference in the positive predictive value of ICA positivity between these two populations. Nevertheless, ICA testing will identify a population who can then be targeted for more rigorous investigation. Genetic testing looking for, for example, non-Asp 57 homozygosity or specific HLA types in nondiabetic related individuals with positive ICA tests raises the positive predictive value of the screen towards levels reached in first degree relatives of Type 1 diabetic patients. Although there are concems about decreasing specificity by multiple tests, this type of stepped approach to screening is likely to be developed in the future.

\section{Is Type 1 diabetes preventable?}

A variety of strategies are being explored for their potential to prevent or interrupt the development of Type 1 diabetes. As mentioned earlier, there are several stages in the prodrome of the disease where intervention might be attempted. Some of the specific approaches under active investigation are described elsewhere in this report (see 'Prevention Trials in Type 1 Diabetes' and "The Glucotoxicity Hypothesis" in "Treatment of Diabetes Mellitus"). Strict glycaemic control and anti-inflammatory and immunosuppressive regimens have been used at the onset of disease and beforehand; anti-oxidant therapy has had some success in disease prevention in animal models; and preliminary studies have been conducted on immunological enhancement and the creation of tolerance to prevent autoimmune destruction of Beta cells.

The ethical issues of disease prevention strategies have already been discussed. They include balancing the benefits of early intervention against the risks of applying 
therapy unnecessarily to some healthy individuals because of the current limitations of disease prediction. For Type 1 diabetes, children are a particularly attractive target for preventive strategies, both because most disease starts in children and because Beta-cell destruction seems to proceed particularly fast in young people. This unfortunate situation makes it easier to evaluate intervention strategies in children. Obviously, children are also a vulnerable group whose protection is most essential. Experience from workers in other diseases affecting children highlight some potential problems.

\section{Lessons from screening programmes for non-diabetic disease}

\section{General lessons}

Certain lessons have been learned over the last decades from screening programmes. In any screening programme, prediction of disease is imperfect. Patients and physicians must interpret complex probabilistic information.

Screening programmes must balance the specificity and the sensitivity of tests. In general, the greater the true positive rate, the greater the false positive rate will be. That is, increased sensitivity leads to decreased specificity. When screening is carried out in populations with low disease prevalence, the false positive rate generally becomes prohibitive.

With particular reference to Type 1 diabetes, the issues of sensitivity and specificity need particular attention. The more tests one performs for predicting that an individual will develop Type 1 diabetes, the more sensitive the battery will be. However, this increased sensitivity comes at the cost of decreased specificity - that is, multiple testing creates a high likelihood of false positives in each testing situation.

Decision analysis techniques have been useful in helping people deal with probabilistic information. In making choices, decision makers must determine what outcomes are possible, how likely each of them are, and how detrimental each of them might be. Decision analysis then requires that decision makers separate choices from chance and assign a value to each possible outcome. Sensitivity analysis can then help determine which factors would change decisions [13]. Professional counselling and assistance with decision making needs further evaluation.

In examining intervention choices for individuals who undergo presymptomatic testing for Type 1 diabetes and who test positive, it is essential to assess their likelihood of developing Type 1 diabetes (i.e. whether the result is a true positive). Such individuals must then evaluate potential treatments, examine the possible side effects of each treatment and estimate the likelihood that the treatment will achieve the desired goal. Currently, few data exist with regard to Type 1 diabetes to support such analyses.

\section{Cautionary lessons}

Screening programmes have been undertaken in the past for a number of diseases, including sickle cell, Tay-Sachs [14], phenylketonuria, congenital hypothyroidism, cystic fibrosis, and Huntington's disease. These programmes were often implemented as soon as a new screening test was developed and prior to demonstrating their safety and benefit [15].

Sickle-cell disease. In the early 1970s, for example, sicklecell screening was mandated in 17 states in the United States. Screening programmes were not accompanied by adequate public or patient education. Misunderstanding of the implications of a positive test for sickle-cell trait led to inappropriate stigmatisation and discrimination in employment, acceptance into the armed forces, and the ability to obtain life insurance [16]. Subsequently, concerns emerged in the black community that trait screening posed significant psychosocial risks [17]. In general, reaction to the sickle-cell programme in the United States was extremely negative. Such a programme might have been improved had there been early attention to public education and patient counselling.

Huntington's disease. Huntington's disease, an incurable autosomal dominant degenerative neurological disorder which manifests in later life, may have particular relevance to prediction of Type 2 (non-insulin-dependent) diabetes mellitus. Conneally has noted the following problems in screening for Huntington's: inadequate quality control of the screening tests may lead to misdiagnosis, with devastating effects on patients and their families; failure to adhere to standard guidelines for pre-test and post-test counselling (a problem which is particularly acute in commercial laboratories which have inadequate counselling); the unnecessary and extreme anxiety and stress that follows a false positive diagnosis [18].

Furthermore, in earlier studies of people at risk for Huntington's disease who were asked whether they would undergo predictive testing if a test became available, the acceptance rate was $70-75 \%[19,20]$. However, now that such a test is available, only $10-15 \%$ of people at risk make themselves available $[21,22]$. This highlights the difficulty of using hypothetical situations to predict peoples' responses in real situations.

Another lesson from the Huntington's experience concerns the conflicting rights of individuals regarding genetic information. Since the Huntington's gene has not been cloned, it is necessary to screen entire families for genetic markers in order to determine any individual's diagnosis. Sometimes, family members prefer not to participate in such screening programmes because they do not wish to know genetic information about themselves. This makes testing of other family members impossible [23]. This situation has direct relevance to Type 1 diabetes, where a single gene is unlikely to be identified as the risk factor. 
Obtaining informed consent for a screening procedure raises other questions. In Huntington's, the genetic tests are truly for presymptomatic diagnosis. No treatment is available to alter the course of the disease. Testing is done primarily to inform patients conceming reproductive decisions or out of the hope that a negative test will allay anxiety. As a result, there is no justification for testing minors who cannot give informed consent. It is hoped that the situation will be different in Type 1 diabetes where potentially preventive intervention is anticipated. Indeed, testing of very young children for presymptomatic Type 1 diabetes should be particularly valuable. This raises ethical considerations concerning surrogate decision making for minors.

The costs of screening for Huntington's are large $(\$ 4,000$ to $\$ 10,000$ per test), partly due to the need for complex genetic analysis of many relatives, and partly because of the costs of expert counselling. In some patients' view, the cost (particularly of counselling, which they did not necessarily want) made the testing prohibitive. It is anticipated that the cost of testing for diabetes will be lower.

Cystic fibrosis (CF). Cystic fibrosis is the most common potentially lethal autosomal recessive disease affecting the white community. The disease is characterised by chronic lung disease and pancreatic insufficiency.

Important lessons can be learned from the experience with screening for $\mathrm{CF}$ reported by Fost [24]. Newborn screening became available in the mid-1980s. Many paediatricians believed that the early aggressive management of minor chest infections would be beneficial. Thus, population screening of newborns was undertaken in some places prior to assessment of the efficacy of early diagnosis in altering the course of the disease.

Concerns arose that early treatment of children with CF might be unnecessary, because many patients with $\mathrm{CF}$ have a benign course. Furthermore, early antibiotic treatment could even be harmful, carrying a risk of developing infection with antibiotic-resistant organisms. False negative results on newborn screening might lead to dangerous complacency in health care providers. Of even more potential relevance to screening for Type 1 diabetes, false positive tests could result in the "vulnerable child syndrome" with adverse effects on the parent/child relationship and the emotional development of the child.

A prospective randomised controlled trial of newborn screening was essential to address these issues [25]. The study raised ethical concerns about withholding diagnostic information from parents of children in the control arm, which were addressed by investigators. Currently, the study has been in progress for 5 years, and so far, no benefits of screening have been demonstrated [26].

\section{Guidelines for the organisation of disease prediction programmes}

From these experiences with other screening programmes, certain general guidelines can be stated:

1. The goals of a screening programme should be clearly defined. Goals could be defined in terms of disease prevention, treatment, or cure.

2. The goals should be demonstrated to be achievable through well-defined pilot studies. Such studies must consider the nature of the disease, technical issues relating to the test procedure, and whether the goals of screening are early therapeutic intervention, improving reproductive decisions, or simply providing information. Application of results from pilot studies must recognise that the positive predictive values of a screening test may vary in different populations.

3. In population screening programmes, initial research must assess the efficacy of the testing process.

4. Adult subjects should be screened only after giving informed consent with adequate pre-testing education and counselling. Post-test counselling should also be available.

5. Minors should be screened only if there is reasonable expectation that a positive test will lead to a beneficial intervention.

6. Good quality control of laboratory tests is essential.

7. Confidentiality of test results should be assured.

8. Costs should be reasonable in relation to benefits.

\section{Ethical issues related to presymptomatic testing and preventive therapy}

Type 1 diabetes is one of the first diseases for which presymptomatic diagnosis and preventive intervention are possible. Nevertheless, the ethical issues raised by presymptomatic diagnosis and preventive treatment for Type 1 diabetes are similar, in many ways, to the issues raised by the other diseases mentioned earlier. Thus, in order to permit research on these aspects of Type 1 diabetes to continue, the cautionary lessons learned from these other programmes should be heeded. There are, however, some important differences between diabetes and these other diseases.

First, there is no definitive predictive test for Type 1 diabetes. This is very different from sickle-cell disease or Tay-Sachs disease [27], where tests exist that can identify carriers with a high degree of certainty. Similarly, although tests for Huntington's disease are not completely accurate, they allow prediction with over 95\% accuracy. In Type 1 diabetes, by contrast, current research is only beginning to refine the accuracy of predictive testing. Thus, the first step in the clinical research agenda must be to study the accuracy of different combinations of tests to predict Type 1 diabetes.

A second difference between Type 1 diabetes and other diseases for which screening programmes have been developed is that one goal of screening for Type 1 diabetes is to prevent the onset of the disease. This effort, requiring clinical trials of interventions to forestall the development of diabetes, raises complex ethical problems. For example, 
it will be difficult to complete studies of predictive testing which require that study subjects be observed until they develop diabetes if, at the same time, parallel studies may offer the possibility of preventing Type 1 diabetes. This will be less of an ethical problem in the earlier studies when interventions are likely to be compared to placebo controls. As soon as an intervention to delay Type 1 diabetes is even partially successful, however, it will be difficult to justify placebo-controlled trials, and difficult to evaluate the accuracy of predictive testing in an untreated population.

A third set of ethical problems will relate to research involving children who are believed to be at increased risk for developing Type 1 diabetes. Because children are regarded as vulnerable subjects, they generally are not eligible for clinical research studies unless the research entails either no risk to the child or the possibility of direct benefit to the child. Population studies of the accuracy of tests to predict who will develop Type 1 diabetes will attempt to develop generalisable knowledge about the immunology and genetics of presymptomatic Type 1 diabetes. Such studies will not be designed to benefit the research subjects directly. Furthermore, these screening studies involve some burdens to the child including both the pain of venipuncture and, more importantly, the psychosocial risks discussed earlier. Some have argued that children should not participate in such studies $[28,29]$, and internal review boards might restrict such studies.

Clearly, studies of the accuracy of predictive testing in children would be more acceptable ethically if they were coupled with a programme of intervention. Predictive testing then would hold the possibility of direct benefit to the child. Depending on the intervention, however, such protocols also may create greater risks for the child. Clinical investigators and internal review boards would have to determine whether the possible risks were justified by the potential benefits. If so, parents will need to be informed of the risks and benefits and then must decide whether to allow their child to participate.

A fourth difference between Type 1 diabetes and other screening programmes relates to the goals of intervention. As noted earlier, in Type 1 diabetes, the ultimate goal would be to prevent the disease. A more realistic interim goal would be to delay the onset of Type 1 diabetes which might result in the following two benefits: it would allow the patient to avoid the medical risks and the lifestyle changes which Type 1 diabetes necessitates; and it might delay the onset of the late sequelae of Type 1 diabetes, such as retinopathy, nephropathy, and peripheral vascular disease.

It will be difficult to evaluate the value of delaying the onset of these late problems, since the experimental treatment will be given now but the effect may not be detectable for 20 or 30 years.

It may seem inappropriate to have a child undergo a burdensome treatment like daily insulin injections in the absence of diabetes, or a potentially dangerous treatment like immunosuppressive therapy, if the therapy only delays the onset of diabetes by 6 months or 1 year. However, most people would consider such therapy acceptable and appropriate if it could delay the onset of blindness or renal failure by 1 year. This may be the net effect of preventive treatment for diabetes, but it is an effect that will be exceedingly difficult to measure.

In deciding whether to undergo such therapies, patients and physicians must choose between a) higher short-term risk associated with the possibility of long-term benefit; and b) lower short-term risk associated with the possibility of lower long-term benefits. This type of choice has been studied in other situations, such as when patients must decide between radiation or surgery for malignancy [30], or when parents consider bone marrow transplantation for their children with sickle-cell disease [31]. The studies show variation among decision makers-some are risk averse, and some are willing to take big risks for the possibility of big benefits. Thus, physicians should be cautious about imposing their own values regarding the risks and benefits of various experimental therapies.

A final set of ethical issues concerns study design. Judgements will have to be made concerning entry criteria and eligibility for studies. At first, studies for presymptomatic testing will focus on first degree relatives of patients with diabetes. More distant relatives, who are also at some increased risk of developing diabetes, may want to participate. As presymptomatic testing becomes more accurate, eligibility criteria for such testing may need to be re-evaluated. Eligibility criteria for therapeutic trials may also be difficult to define. Results from presymptomatic tests will generate a probability that a particular individual will go on to develop diabetes. People with a high probability of developing diabetes will have a more advanced pre-diabetic state and may not respond to preventive therapy. People with a low likelihood of developing diabetes will not need preventive therapy. Judging where to set the eligibility criteria will be as much an ethical as a scientific decision.

\section{Behavioural/psychosocial issues}

Because these studies examine a new type of medical information and because they necessarily involve families, rather than simply individual patients, careful attention to behavioural and psychosocial issues will be necessary. Studies should examine the psychological effect of presymptomatic testing. Patterns of family communication about disease risk should be investigated. Careful attention to informed consent for research will be important.

\section{Economic issues}

The prevention of diabetes would obviously have 
enormous economic benefits. However, initial efforts at prevention may merely lead to a temporary delay in onset. The costs of patient identification and intervention using different strategies should be compared with the likely savings that will result.

\section{Conclusions and recommendations}

- By using various combinations of immune markers and metabolic testing, we are now able to predict with a reasonable degree of certainty which first degree relatives of a child with Type 1 diabetes will develop Type 1 diabetes. This predictive ability enables us to define a cohort that could be enrolled in clinical trials of presymptomatic intervention. Such studies should be endorsed and funded.

- Genetic markers currently have a limited role in diabetes prediction.

- Although diabetes prediction appears to be possible in the general population using current techniques, the predictive power of such tests is low and the economic and social costs of identifying individuals at risk is high. Consequently, it is our view that intervention strategies should at present be reserved for first degree relatives of Type 1 diabetic probands.

- We endorse widespread screening of families with Type 1 diabetic members to establish the prevalence of known risk markers, to establish the prognostic significance of novel markers, and to identify high-risk individuals for intervention trials. Family screening for Type 1 diabetes markers should only be undertaken in the context of wellprepared research protocols. Pre- and post-test counselling and psychological support should be available to individuals who are screened.

- Intervention studies designed to delay or prevent the onset of Type 1 diabetes should only be attempted in the context of approved prospective controlled trials. They must have the statistical power to reach a conclusive outcome. Their risk-benefit ratio should be acceptable to participants, their families, and to the investigators and the internal review boards.

- Those planning or undertaking intervention trials should do so as far as possible in a coordinated fashion to avoid unnecessary competition or duplication of results. Registries of such screening programmes and interventions should be maintained, with continuing efforts to improve standardisation of predictive markers and outcome measures.

- Because of the complexity of the decisions which patients or parents must make, research should be conducted on ways to help individuals understand information and make informed choices about early detection and presymptomatic interventions. Such research should focus on:

- the risk-benefit ratio of the early detection programme as perceived by the childand family.
- the positive and negative effects of early detection on the child and family's a) functional state and well-being; b) participation in treatment programmes; c) general attitude to Type 1 diabetes; and d) ability to avoid discrimination and stigmatisation in education, employment, and in obtaining insurance.

- It should be recognised that both early detection programmes for Type 1 diabetes and clinical trials of presymptomatic interventions raise serious ethical and policy issues. Other screening programmes (for example, those for sickle-cell disease and Huntington's disease) have been substantially undermined because of failure to address these ethical and policy issues in advance. Therefore, in order to achieve desired clinical and scientific goals, it is essential for the diabetes community to address these issues in advance. Research in clinical ethics and health policy relating to diabetes should be encouraged and supported. As an example, we recommend a central registry to record adverse personal, social, and economic consequences of Type 1 diabetes prediction programmes and presymptomatic prevention programmes. This registry would be unique, but could serve as a model for many other disease screening efforts including those related to the genome project.

- This work group wishes to encourage the JDF to exert its influence on national and international funding agencies to support major research initiatives into both the scientific and ethical issues associated with early detection and preventive treatment programmes designed to cure Type 1 diabetes.

Acknowledgements. This report is the result of the Work Group 8: Consequences of Disease Prediction at the Third JDF World Conference on Diabetes in Monte Carlo, Monaco, 8-10 March 1992. Chaiman: M. Siegler. Work group participants: P.M. Conneally ${ }^{1}$, N. Fost ${ }^{2}$, E.A.M. Gale $^{3}$, M. M. Kaback ${ }^{4}$, J. Lantos, N. Maclaren', S.G. Pauker ${ }^{6}$, J.A. Robertson ${ }^{7}$, M.A. Rothstein ${ }^{8}$. Rapporteur: S. Amiel.

${ }^{1}$ Indiana University, Indianapolis, Indiana, USA; ${ }^{2}$ Department of Pediatrics, University of Wisconsin, Madison, Wisconsin, USA; ${ }^{3}$ Department of Diabetes and Metabolism, St. Bartholomew's Hospital, London, UK; ${ }^{4}$ School of Medicine, University of Califomia, San Diego, California, USA; ${ }^{5}$ Department of Pathology and Laboratory Medicine, College of Medicine, University of Florida, Gainesville, Florida, USA; Wew England Medical Center, Boston, Massachusetts, USA; ${ }^{7}$ School of Law, University of Texas, Austin, Texas, USA; ${ }^{8}$ Health Law and Policy Institute, University of Houston, Houston, Texas, USA.

\section{References}

1. Lappe M, Gustafson J, Robbin R (1972) Ethical and social issues in screening for genetic disease. N Engl J Med 285: 1129-1132

2. Rothstein MA (1989) Medical screening and the employee health cost crisis. The Bureau of National Affairs, Washington, DC

3. Tam AC, Smith CP, Spencer KM, Bottazzo GF, Gale EAM (1987) Type 1 (insulin-dependent) diabetes: a disease of slow clinical onset? Br Med J 294: 342-345

4. Gorsuch AN, Spencer KM, Lister J et al. (1981) The natural history 
of type 1 (insulin-dependent) diabetes mellitus: evidence of a long pre-diabetic prodrome. Lancet II: 1363-1365

5. Chase HP, Voss MA, Butler-Simon N, Hoops S, O'Brien D, Dobersen MJ (1987) Diagnosis of pre-type 1 diabetes. J Ped 111: 807-812

6. Vardi P, Crisa L, Jackson RA (1991) Predictive value of intravenous glucose tolerance test insulin secretion less than or greater than the first percentile in islet cell antibody positive relatives of type 1 (insulin-dependent) diabetic patients. Diabetologia 34: 93-102

7. Dorman IS, Trucco M (1989) How much does HLA-DQ phenotype contribute to the incidence of type 1 diabetes in Allegheny County, PA? Diabetes 38 [Suppl 2]: 34A

8. Faustman D, Eisenbarth GS, Daley J, Breutmeyer J (1989) Abnormal T-lymphocyte subsets in type 1 diabetes. Diabetes 38: 1462-1468

9. Bonifacio E, Bingley PJ, Dean BM, Shattock M, Dunger D, Gale EAM (1990) Quantification of islet cell antibodies and prediction of insulin-dependent diabetes. Lancet 335: 147-149

10. Riley WJ, Maclaren NK, Krischer J et al. (1990) A prospective study of the development of diabetes in relatives of patients with insulindependent diabetes. New Engl J Med 323: 1167-1172

11. Colman PG, Eisenbarth GS (1988) Immunology of type 1 diabe-tes. In: Alberti KGMM, Krall LP (eds) The diabetes annual/4. Elsevier, Amsterdam, pp 17-45

12. Bingley PJ, Shattock M, Chusney $G$ et al. (1991) Unexpectedly high prevalence of islet cell antibodies in healthy children aged 8-13 Diabetic Med 8 [Suppl 1]: A3

13. Pauker SG, Kassirer JP (1987) Decision analysis. N Engl J Med 316: 250-258

14. Childs B, Gordis F, Kaback MM, Kazazian HG (1976) Tay-Sachs screening: motives for participating and knowledge of genetics and probability. Am J Hum Genet 28: 537-549

15. Fletcher JC (1984) Ethical and social aspects of risk predictions. Clin Genet 25: 25-32

16. Motulsky G (1989) Societal problems in human and medical genetics. Genome 31: 870-875

17. Beutler E, Boggs DR, Heller P et al. (1971) Hazards of indiscriminate screening for sickling. N Engl J Med 285: 1485-1486
18. Jenkins JB, Conneally PM (1989) The paradigm of Huntington's disease. Am J Hum Genet 45: 169-175

19. Markel DS, Young AB, Penney JB (1987) At-risk persons' attitudes toward presymptomatic and prenatal testing of Huntington's disease in Michigan. Am J Med Genet 26: 295-305

20. Meissen GJ, Berchek RL (1987) Intended use of predictive testing by those at risk for Huntington's disease. Am J Med Genet 26: 283293

21. Quaid KA, Brandt J, Faden RR, Folstein SE (1989) Knowledge, attitude, and the decision to be tested for Huntington's disease. Clin Genet 36: 431-438

22. Craufurd D, Dodge A, Kerzin-Storrar L, Harris R (1989) Uptake of presymptomatic predictive testing for Huntington's disease. Lancet II: 603-605

23. Shaw MW (1987) Testing for the Huntington gene: a right not to know, a right to know, or a duty to know. Am J Med Genet 26: 243-246

24. Wilfond BS, Fost $N$ (1990) The cystic fibrosis gene: medical and social implications for heterozygote detection. JAMA 263: 2777. 2783

25. Roberts L (1990) To test or not to test. Science 247: 17-19

26. Caskey CT, Kaback MM, Beaudet AL (1990) The American Society of Human Genetics statement on cystic fibrosis. Am J Hum Genet 46: 393

27. Pauker SG, Pauker SP (1987) Prescriptive models to support decision making in genetics. Birth Defects 23: 279-296

28. Fletcher JC (1984) Ethical and social aspects of risk predictions. Clin Genet 25: 25-32

29. Motulsky AG (1989) Societal problems in human and medical genetics. Genome 31: 870-875

30. McNeil BJ, Weichselbaum R, Pauker SG (1981) Speech and survival: tradeoffs between quality and quantity of life in laryngeal cancer. N Engl J Med 305: 982-987

31. Kodish E, Lantos J, Stocking C, Singer PA, Siegler M, Johnson FL. (1991) Bone marrow transplantation for sickle cell disease: a study of parents' decisions. N Engl J Med 325: 1349-1353 